\title{
Changing Demography of Pancreatitis Patients in India - A Hospital Based Study
}

\author{
Vaidya Balendu Prakash ${ }^{1 *}$, Megha Prakash¹, Vaidya Shikha Prakash ${ }^{2}$, Sneha Tiwari ${ }^{1}$, Shakshi Sharma ${ }^{2}$, Vaidya \\ Pooja Jaryal ${ }^{2}$ and Jyoti Tewari ${ }^{1}$ \\ ${ }^{1}$ VCPC Research Foundation, Uttarakhand, India \\ ${ }^{2}$ Padaav - Speciality Ayurvedic Treatment Centre, Uttarakhand, India \\ *Corresponding Author: Vaidya Balendu Prakash, VCPC Research Foundation, Uttarakhand, India.
}

Received: July 25, 2019; Published: August 12, 2019

\begin{abstract}
Pancreatitis is a disease of inflammation of the pancreas and is rising exponentially in the world. Different causes are attributed to the disease with alcohol, tobacco and family history as the major causes. However, its etiology largely remains unknown. It is reported that the Southern states of India have highest incidences of Pancreatitis, ranging from 114-200/100,000 population. Rest of the globe reports 1.6-27 cases per 100,000 populations. A North India based ayurvedic clinic has collected details of age, sex, religion, caste, profession, native place, dietary habits and family history from 800 well-diagnosed patients of Recurrent Acute/Chronic Pancreatitis (RA/CP), enrolled from January 1997 to June 2019. Surprisingly, patients from the Northern states of India outnumber those from the Southern states. Similarly, incidences of Pancreatitis were significantly higher among non-alcoholics, non-tobacco users and those with no previous family history. The male-female ratio was 4.6:1. Highest incidences were noted in the age group of nineteen to forty five years. Interestingly, highest cases of Pancreatitis were reported among the service class and students. A comprehensive survey needs to be carried to understand the magnitude of Pancreatitis in this region. An emphasis should also be made to explore more etiological factors.
\end{abstract}

Keywords: Pancreatitis; Recurrent; Acute; Demography

\section{Introduction}

Pancreatitis, the inflammatory disorder of the pancreas, is characterized by moderate to severe abdominal pain, nausea, vomiting, indigestion, weight loss and steatorrhea, affecting individual's personal, professional and social life [1]. The first case of Acute Pancreatitis (AP) was reported in year 1652 by Dutch anatomist Nicholas Tulp [2]. Later, Reginald Fitz, a pathologist from Harvard, in 1889, established the diagnosis of Acute Pancreatitis by specifying its signs and symptoms [3]. Hans Chiari of Austria related Pancreatitis to auto-digestion of pancreatic tissues (1896). Chronic Pancreatitis (CP) was ascribed in 1946 by Comfort, an American anatomist, who also highlighted its possible causes [4].

Henceforth, there has been a rising trend in the incidences of Pancreatitis across the world. The incidences of Acute Pancreatitis in Wales, UK rose from 27.6 per 100,000 populations in 1999 to 35.9 in 2010 with an annual increase of $2.7 \%$ per annum [5]. A nationwide hospital based study in Columbus recorded a $13.3 \%$ rise in Acute Pancreatitis related admissions from 2002-05 to 2009-12 [6]. A field study carried in 1993 in Kerala, a Southern state of India, reported highest incidences of tropical Chronic Pancreatitis in the world (up to 126 per 100,000 population) [7].
Chronic Pancreatitis also showed rising trend from 2.94 in 197786 to 4.35 in 1997-2000 per 100,000 populations in a population based study in Olmsted County [8]. The trend was also reported from China where prevalence of CP rose from 3.1/100,000 in 1996 to $13.5 / 100,000$ population in 2003 [9]. Similarly, high prevalence of tropical Chronic Pancreatitis has been reported from Southern India (114-200/100,000 populations) in 2004 [10]. Japan carried seven nationwide surveys between 1994 to 2011 regarding the epidemiology of Pancreatitis and demonstrated increasing prevalence of CP from 28.5/100,000 in 1994 to 52.4/100,000 in 2011 [9]. A South India based tertiary care centre reported $13.3 \%$ rise from 2000-06 to 2007-13 [11]. Global Burden of Disease study of 2015 has reported 8.9 million cases of Pancreatitis in the world with 132,700 deaths [12].

Alcohol is considered the most common cause of CP/AP other than gall stones and genetic or environmental factors. However, there are fair number of cases who have never consumed alcohol in their lifetime. But there is no doubt that alcohol increases the sensitivity of the pancreas and the risk of Pancreatitis is undoubtedly high among alcoholics. Similarly, the risk of Pancreatitis was observed to be higher among smokers in comparison to non-smokers 
but could not be attributed as the cause of CP/AP [13]. The role of dietary factors in the etiology of Pancreatitis is still unclear. Heredity is also a causative factor of Pancreatitis and such patients could be placed among high risk of developing pancreatic cancer, ranging from $40-55 \%$. The disease is more common among men and affects middle aged individuals the most. In recent years, there are reports about its increasing trend in paediatric population as well [13].

The presented study was carried to understand the various aspects of Pancreatitis.

\section{Methodology}

A North India based hospital, practicing Ayurveda, has been receiving patients of different variants of Pancreatitis since 1997 [14]. Fifty cases were enrolled from 1997 to 2013. However, the number increased multi-fold owing to a public event held in 2014 in the presence of policy makers, professionals, scientists and media. Till date, 800 patients have been enrolled at this clinic. All these patients had been diagnosed by the subject experts at distinguished hospitals for different variants of Pancreatitis (Figure 1a) belonging to both sexes (Figure 1b) from age group ranging from 3 to 72 years with a mean age of $30.07 \pm 11.3$ years(Figure 1 c) and had a history of disease ranging from 1 month to 25 years (Figure 1d). These patients were interviewed for the onset of symptoms, diagnostic hospitals, emergency attacks/hospitalizations and use of enzymes, along with their personal profile including dietary habits, native place, education, profession and family history. A baseline blood investigations, consisting of hemogram, lipid, liver, kidney and diabetic profile, Serum Amylase, Lipase, Vitamin B12 and D3 were carried to understand the status of the patient prior to the commencement of Ayurvedic treatment. A detailed data of these patients regarding their age, native place, food habits, education, profession and family history was collected at enrolment.

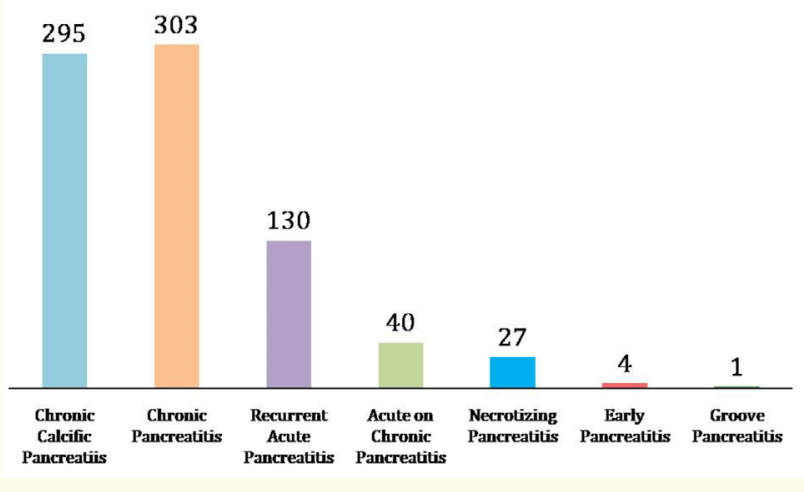

Figure 1a

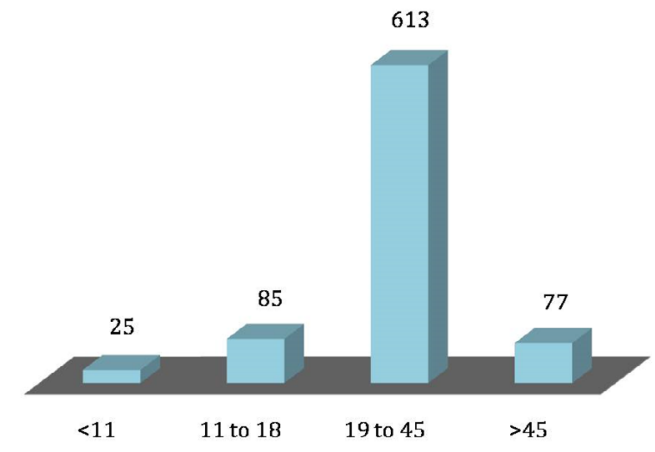

Figure 1c

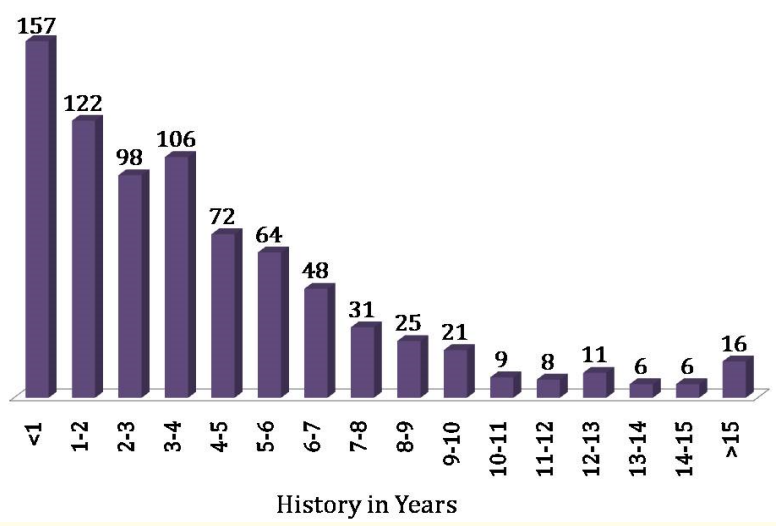

Figure 1c

Outcomes

- Incidences linked with risk factors: It was found that incidences of RA/CP are more prevalent among non alcoholics (69\%), non tobacco users (80\%) and those with no family history (96\%) (Figure $2 \mathrm{a}, \mathrm{b}, \mathrm{c}$ ). 
- Geographical distribution: North India reports higher incidences of RA/CP in comparison to South India (Figure $3 \mathrm{a}, \mathrm{b}$ ). These observations might be non-conclusive but one thing is evident that incidences RA/CP are widely distributed in the country.

- Vitamin D deficiency: $87.58 \%$ patients were found to be deficient in Vitamin D3 (n=404/800) (Figure 4).

- Profession and education wise distribution: The incidences were more prevalent among service class and students (Figure 5). Maximum number of patients were from engineering background.

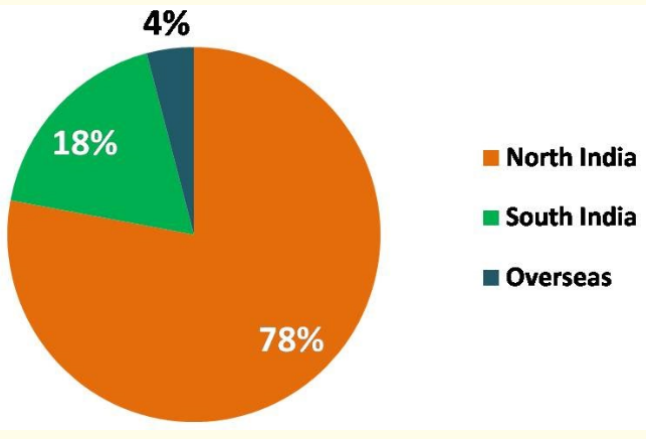

Figure 3a

\begin{tabular}{|c|c|c|c|}
\hline Uttar Pradesh & 122 & Andhra Pradesh & 20 \\
\hline Maharashtra & 69 & Assam & 18 \\
\hline Delhi & 68 & Tamil Nadu & 16 \\
\hline Karnataka & $\mathbf{5 9}$ & Bihar & 16 \\
\hline Gujarat & 51 & J \& K & $\mathbf{8}$ \\
\hline Uttarakhand & 47 & Odisha & $\mathbf{8}$ \\
\hline Haryana & 47 & Himachal Pradesh & 7 \\
\hline Rajasthan & 37 & Jharkhand & $\mathbf{5}$ \\
\hline Madhya Pradesh & 32 & Tripura & $\mathbf{2}$ \\
\hline Telangana & 31 & Goa & $\mathbf{2}$ \\
\hline West Bengal & 27 & Lakshadweep & 1 \\
\hline Kerala & 24 & Sikkim & 1 \\
\hline Chhattisgarh & 24 & Chandigarh & 1 \\
\hline Punjab & 23 & Overseas & $\mathbf{3 4}$ \\
\hline
\end{tabular}

Figure 3b

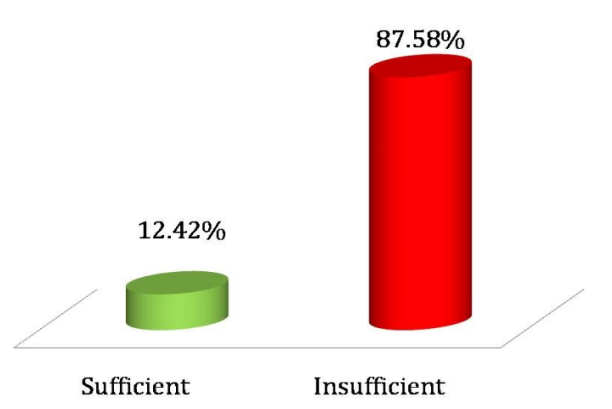

Figure 4

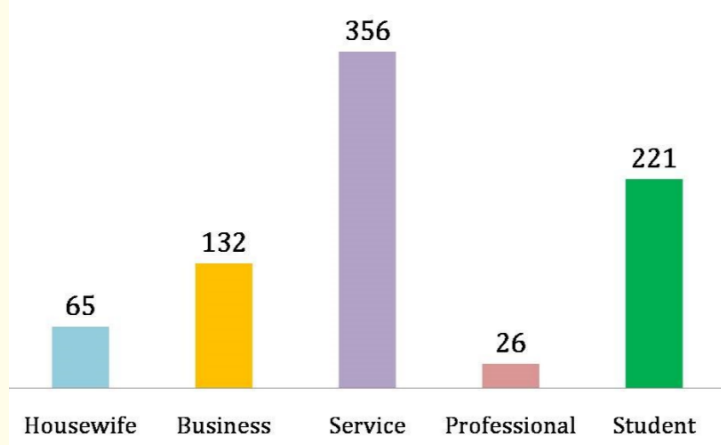

Figure 5 


\section{Discussion}

Pancreatitis, which was first reported in seventeenth century, now affects nearly $0.15 \%$ of the world population with high morbidity rate. 55\% patients die within 20 years of diagnosis [15]. Alcohol, tobacco and genetic factors have been identified for aggravating RA/CP. However, no confirmatory causative factor is yet established. The disease has rising trend and incidences are being reported from various parts of the world.

In the present study, a physician of Ayurvedic medicine (Complementary and Alternative Medicine) situated $250 \mathrm{kms}$ north east from New Delhi has reported demography of its 800 patients, spread over 22 years duration. The observation brings some interesting findings as incidences are reported more among nonalcoholic and non-tobacco users and those with no family history. This is the first study of its type where RA/CP patients from North India outnumber South Indian patients. One may state that this number could be due to the presence of the clinic in North India. However, the second highest number of patients come from Maharashtra, which is far away from this clinic, nulling the aforesaid statement. The results clearly indicate that the incidences of RA/ $\mathrm{CP}$ are spread all over the country. The findings also bring interesting information about professional and educational background of patients. The high incidences in service class and students might be indicative of a linkage between stress and RA/CP.

The study is in equivalence to the earlier studies that state that the incidences are more common in males and in productive age group [16]. However, there are rising cases of paediatric Pancreatitis [13]. The study does not draw any conclusion but might create a pathway for mapping of Indian population for the incidences of $\mathrm{RA} / \mathrm{CP}$ and developing hypothesis for a causative factor.

\section{Conclusion}

The incidences of RA/CP are rising in India and are not restricted to one region only. The disease should be understood well to reduce their burden on individuals and their families.

\section{Acknowledgement}

Authors are thankful to all the patients and their families for sharing their details.

\section{Conflict of Interest}

Authors declare no conflict of interest.

\section{Bibliography}

1. Torpy JM., et al. "Pancreatitis". Journal of the American Medical Association 307.14 (2012): 1542.

2. Pannala R.. et al. "Acute Pancreatitis - A Historical Perspective". Pancreas 38.14 (2009): 355-364.

3. Busnardo AC., et al. "History of the pancreas". The American Journal of Surgery 146.5 (1983): 539-550.
4. Rustgi AK. "A Historical Perspective on Clinical Advances in Pancreatic Diseases". Gastroenterology 144 (2013): 12491251.

5. Roberts E., et al. "The incidence of acute pancreatitis: impact of social deprivation, alcohol consumption, seasonal and demographic factors". Alimentary pharmacology and therapeutics 38.5 (2013): 539-548.

6. Krishna SG., et al. "The changing epidemiology of Acute Pancreatitis hospitalizations: A decade of trends and the impact of Chronic Pancreatitis". Pancreas 46.4 (2017): 1.

7. Tandon RK., et al. "Chronic pancreatitis: Asia-Pacific consensus report". Journal of Gastroenterology and Hepatology 17 (2002): 508-518.

8. Lew D., et al. "Chronic Pancreatitis: Current Status and Challenges for Prevention and Treatment". Digestive Diseases and Sciences 62.7 (2017): 1702-1712.

9. Yadav D., et al. "Incidence, prevalence, and survival of chronic pancreatitis: a population-based study". American Journal of Gastroenterology 106 (2011): 2192-2199.

10. Garg PK and Tandon RK. "Survey on chronic pancreatitis in the Asia-Pacific region". Journal of Gastroenterology aHepatology 19 (2004): 998-1004.

11. Rajesh G., et al. "Time trends in the etiology of chronic pancreatitis in South India". Tropical Gastroenterology 35.3 (2014): 164-167.

12. GBD 2015 Disease and Injury Incidence and Prevalence, Collaborators. (8 October 2016). "Global, regional, and national incidence, prevalence, and years lived with disability for 310 diseases and injuries, 1990-2015: a systematic analysis for the Global Burden of Disease Study 2015". Lancet. 388 (10053): 1545-1602

13. Yadav D., et al. "The epidemiology of Pancreatitis and Pancreatic Cancer". Gastroenterology 144.6 (2013): 1252-1261.

14. Jaiswal YS., et al. "A glimpse of Ayurveda - The forgotten history of principles of traditional medicine". Journal of Traditional and Complementary Medicine 7 (2017): 50-53.

15. Lowenfels AB., et al. "Prognosis of chronic pancreatitis: an international multicenter study". American Journal of Gastroenterology 89.9 (1994): 1467-1471.

16. Sawant P., et al. "Chronic Pancreatitis - Indian Scenario". Medicine Update (2005): 418-424.

\section{Volume 2 Issue 7 September 2019 (C) All rights are reserved by Vaidya Balendu Prakash., et al.}

